http://dx.doi.org/10.7494/automat.2013.17.2.173

Ewa Dudek-Dyduch*, Krzysztof Rączka*

\title{
Selection of Databases \\ for Real Estate Information Systems
}

\section{Introduction}

This article applies to a specific type of information systems, namely systems for the analysis of real estate market and real estate evaluation. These systems have special properties, which cause that the system performance depends strongly on the type of database that is used.

One of the key stages in creating an information system for real estate evaluation is to choose the right type of database. Co-author implemented a prototype system, called Pro/Rzeczonawca, which works in practice. This article presents considerations that led to the proposal of the concept of two different systems working together and to the selection of the appropriate type of database for each of them.

The real estate market in Poland is in phase of a very dynamic growth and change. On one hand it is forced by customers and on the other by the greater opportunities of access to any information directly or indirectly related to the property market. However, there is a lack of information systems supportingthe service (among the others analysis) of real estate market, on a broader level than supporting only a single issue or a narrow area of topics, for example, only the real estate agent or just real estate appraiser. The main objective of hitherto created systems is to monitor the value of the property in the area covered by the analysis. Currently in Poland, there are several systems such as Walor [18], EWycena [20], E-Rzeczoznawca [19], which monitor the real estate transaction prices and rents for professional appraisers. The disadvantage of these systems is that only local areas are monitored, and systems do not cooperate in the exchange of data. Another disadvantage is the possibility just to monitor the value of the property, without being able to analyze collected data. With a large number of collected data, the system becomes useless because without algorithms for analyzing the data in the database, a person is not able to draw any conclusions about the monitored area of real estate market.

* AGH University of Science and Technology, Krakow, Poland 
Before proceeding to real estate valuation, expert performs a thorough analysis of assessed property using available sources of information, such as records of transactions, market analysis and site visits [1]. The result is that the real estate market analysis and valuation of real estate is very complicated, moreover, often do not have access to reliable data [2].

It follows from the above that there is a need to create information systems to support the work of appraisers and real estate brokers. These systems will be called by the authors as information REM systems (Systems for Real Estate Market).

These tools will be database applications. The first step in the establishment of systems is the selection of appropriate types of databases.

Objective of this article is threefold:

- presentation of the required characteristics of information systems for real estate valuation, relevant to the selection of databases,

- proposing the concept of cooperating two types of systems with different scope of tasks,

- analyzing the existing types of databases in terms of the real estate evaluation systems and database types to select the most appropriate.

There are many publications concerning the comparison of properties of different types of databases, among others [3, 4, 5]. However, the comparisons of these analyze mainly technical properties of database and do not include properties arising from specific applications.

The article presents successively:

- the concept of two different types of systems for the real estate market experts,

- the specific properties of information stored and used in REM systems databases

- analysis of different types of databases that can potentially be used while creating the REM system.

\section{Information systems for an analysis of the real estate market and property valuation}

Below will be presented the concept of two different systems for the real estate market along with their brief characteristics. This division is important to select the correct type of databases.

\subsection{Disadvantages of currently implemented property valuation}

REM systems are designed for the real estate market experts such as appraisers and real estate agents, state institutions, individuals and other institutions interested in reliable information about the current situation in the real estate market. 
The result of the work of real estate expert is so called appraisal report consisting of two parts: the valuation of the property with its grounds and formal part containing among other things the legal basis and the legal status of the property being valued.

The traditional way of property valuation makes accurate determination of its value and objectification process of estimating the property impossible. It consists of the following steps:

1. analysis and characterization of traits of estimated property

2. analysis of the local market of ,similar" properties

3. comparing"similar" properties to the property which is valued

4. determination of the value of the property being estimated

5. verification of the results and a possible return to the first stage

6. valuation reason.

The first three stages, and the fifth stage is based on the personal experience of an expert who has analyzed a large number of incomplete and uncertain data. As a result of further analysis the expert determines the appropriate parameters. Only for the fourth stage areknown simple (very primitive), approximate formulas. They use the parameters defined in the earlier stages. Most calculations are carried out using Excel or a calculator. Therefore, to specify the value of the property has a large impact of the expert's subjectivity. What's more, experts focusing on crudely executed, time-consuming calculations (step 4) neglect the analysis which ensure the proper determination of the parameters. In addition, the primitive formulas may not include all the information affecting the value of the property.

Currently, Poland real estate expert usually only uses their own materials (information about the properties and expertise), and uses only his experience. There is no access to information held by other experts and, consequently, do not have access to global data on the real estate market in the country.

\subsection{Proposal of automation of real estate expert work automation}

To make the situation better, it is necessary to create information systems that support and automate all stages of the work of an expert.

The following are the basic tasks to be carried out by modern systems of REM:

a) Collection of very large amounts of data about real estates and transactions. These are the descriptions of the properties, their photos, descriptions of transactions, expertise along with their justifications, and the entire appraisal. It should be noted that these figures are prepared in the inconsistent way by various experts, they are often unreliable and incomplete.

b) Making various types of analyzes aimed at determining the relevant parameters. It must be all those parameters that an expert previously designated for subsequent stages 1-3 and 5, furthermore there may be additional parameters. To perform analysis auto-select option of "similar" properties as well as the possibility of selection made by the expert should be implemented. 
c) Making the valuation using the new, more advanced methods, which use more parameters and other information [2]. The system should as far as it ispossible automate all processes which flow is clearly defined.

d) Supporting the process of creation of a justification for expertise that has been made (in part automatically).

In addition, the system should have a module that allows you to build a knowledge base on the basis of the experience of many experts. It should be emphasized that an important feature of the system must be flexibility involving the possibility of using the experts' experience at every level of expertise.

The expert valuation should be based on data from the local real estate market. It is often not possible for two reasons:

a) the absence of representative transactions in the underdeveloped local market (eg. in rural areas),

b) lack of access to all transactions for large quantities (eg. in the city).

In this case, the information from another comparable property market should be used. Unfortunately the lack of database of real estate transactions prevents such a solution.

To solve this problem, the authors propose the use of two different systems to fully support, due process of property valuation. These are:

- a system of monitoring and analyzing the overall situation in the real estate market (M-A System),

- an expert system that is designed for the local market analysis and valuation of real estate in the local market (REE system - Real Estate Expert System).

The M-A system should be a multi-access system, supplied with the information on real estate transactions by all experts. At the same time it can be used by even hundreds of experts. Whereas, REE system is designed for individual office expert and contains only his data.

The M-A systemshould therefore be created in the web version and be available online. It should allow to collect the following information:

- the real estate market covering the whole country: the price of real estate transactions, the bidding data and data on rents,

- socio-economic data affecting the real estate market.

On this basis, the system should perform advanced global analysis. This system could be used not only by experts but also by ordinary citizens.

REE system should be created in the standalone version (allow for an offline access). Its intended purpose should be advanced analysis first of all local real estate market and supporting all stages of expert's work (valuation and its justification). In the expert's office the system can be used in parallel maximum by a few users. In the absence of a representative, or complete data from the local market expert can connect to the M-A system. 
This way he will simply get the missing data, or data on transactions with "similar" local markets. In addition, the system can use the M-A system in order to verify the established evaluation report.

Both systems should be able to visualize the property on the map, so they must be integrated with GIS (Geographic Information System).

\subsection{Characteristics of data on real estate transactions}

This section will present the specific properties of stored and used information in the databases of both types of systems. The data in the systems will be collected over a long period of time. Data structures used for description of particular transaction can differ and dynamically change over time.

Characteristics of information stored in databases systems REE:

- information about the real estate market stored in the database, as well as performed analysis and opinions, will have the form of documents,

- documents saved in the database will have a complex character which will have only partially systematic structure,

- there is not very large amount of data, due to the local nature of the system - within one office (or office network).

Characteristics of information stored in the database of M-A system:

- information about the real estate marketstored in the database will be less textual than in the REE,

- the structure of the data collected will vary over time and will be developed, due to the different description of the market data,

- a very large amount of data - the data will be collected by a large number of experts working independently of each other.

In conclusion, the fundamental criteria for selecting the appropriate database for these systems are the following:

- REE system: the ability to collect complex documents that have only partially systematic structure, and easy search requested information from them,

- M-A system: the ability to collect a very large amount of data, the speed of access to stored data, the possibility of rapid changes in the structure of collected documents, the freedom to add other types of documents; all of these can change the structure of the database.

Due to the necessity of integration of these two systems with GIS you should analyze the representation of the data reported to the GIS system and how it is stored in a GIS. The need to analyze results from a very large amount of data collected by the GIS. There should be a selection of the database systems that allow for fast processing of large amounts of data. 
There should be paid special attention to other, apart from the features mentioned above,which the real estate market is characterized by, such as:

- uncertainty of the data relating to real estate transactions, often resulting in necessity of supplement or modification of the information,

- individualism of experts' assessments, resulting in different and individual ways of data recording,

- a variable ways of drawing conclusions about the present and the future based on historical data, by various experts, what makes it necessary to archive large amounts of data on transactions, particularly in M-A systems,

- taking into account the real estate market linkage with socio-economic situation of the monitored area, resulting in necessity of storage information about the unknown and the 'a priori structure

- the need to store the data which are often defined by erroneous and contradictory laws.

These properties make that structure of the stored information is not fixed and known in advance. This forces necessity of a flexible and extensible data structure.Type of the database must provide the ease of changing the data structure.

Additionally, you should take into account the fact that the input data may contain errors due to human factors. There should be developed sophisticated mechanisms to control the correctness of the entered data into the database for systems of this type. Developed mechanisms should take into account the specifics of obtaining and entering data into the database, and specific data on the real estate market.

The experience of the authors suggests that we should consider the possibility of choosing another type of database system for REE system and another for M-A system due to the different nature of these systems.

\section{Analysis of types of databases for REM systems}

In this chapter we will examine the types of databases, due to the possible use of the REM system. The result of performed characteristic will be further comparison between the characteristics of each individual database types and the choice of the most suitable for REE systems and the MA system.

In recent years the scope of the use databases was significantly expanded. There are still new areas where there is a necessity of storing, searching and analyzing data. There are still new problems related to the effectiveness of the operation of databases in a changing environment. The new requirements led to the development of new database systems and the need to develop new methods for searching and analyzing the data collected. Initially opportunity to search data that meets certain criteria was sufficient. Then there was taken into consideration the problem of data mining i.e. the acquisition of knowledge on the basis of the collected data, the discovery of relationships that are not explicitly stored in the database. On the other hand, a large amount of information in databases drew attention 
to efficiency problems. As a result, new types of databases have been created, NoSQL databases recently. NoSQL databases are very efficient, which is crucial in applications that use large amounts of data [3].

\subsection{Relational databases}

This section examines the advantages and disadvantages of using relational databases in REM systems.

Referential integrity in relational databases are one of the most important characteristics of REE systems. Their existence is very important in the context of complex documents. One document can be stored even in several tables. The high level of normalization of the database increases the security and integrity of data. The disadvantage of normalizationis a decline in performance of database operations. In systems using REM the best usage of these properties would be to create such a database structure that is at the average level of normalization(this practice is also used in most commercial systems). High efficiency of activity should be kept, but also eliminate the places that could potentially lead to data inconsistency.

Another important feature of relational databases is transactionality. The conditions fulfilled by transactions are precisely described by ACID rules. ACID rules in relational databases, ensure their reliability [6]. For REE systems transactionality of database is a very important feature for two main reasons. Firstly large documents with developed structure are recorded what causes the performance of many SQL language instructions, secondly, documents are edited by many users simultaneously. Thus REE system should provide the ability to create complex documents which different parts can be edited at the same time by several users. The creation of such a functionality without transaction mechanism would be impossible.

Language for relational databases is SQL. Using SQL is convenient for structured data [3]. The usage of this language for complicated data is difficult because it is designed for structured data and stored in tables of rational organized databases [3]. Because the data in the REM system have not uniform structure, so the use of the SQL language is not comfortable here.

The main disadvantage of relational database systems is to reduce the reduce range of applications. Inside each tuple contents of each attribute must be an atomic value. The result is that it is difficult to model the data, which structure is not defined in an unambiguous manner, and as it is often inthe REM system. Information referring to the single object such as a document are stored in different tables, so that modeled data are not consistent representation of a real object. Another disadvantage resulting from the above-mentioned limitations is that not all data which are supposed to be stored in the database can easily be stored in tables. This causes that the database structure is complicated, difficult to maintain and not very efficient. 
REM systems will store a very large amount of data. Commonly used nowadays relational databases can handle a large amount of data, but the cost of maintenance in this case is much higher than in the case of smaller data sets. In relational databases, the largest cost (financial) generates a hardware infrastructure and the execution time of some operations such as searching and retrieving of data. Relational databases can be scaled by running them on a better server [3]. They do not work easily in a distributed architecture, because joining tables across distributed systems is difficult [3].

\section{Mechanism of ORM (Object-Relational Mapping)}

Below we will present the considerations on the use of ORM mechanism in the REM systems.

The ORM mechanism is the mapping of data from object model to incompatible relational model. As a result we get an additional layer of access to data stored in the database, which reflects the modeled objects. In the REM system it frequently happens that one object (document) has an attribute which is a list of elements. This object is stored in a relational database in several tables. A programmer wanting to add the object to the database using SQLlanguage, must perform several queries. Instead of them, ORM mechanism transform object to model created by means tables. There is a conversion of the object which has been created in database by using the tables of the model which is mapping the actual form. ORM causes that the programmer do not use the SQL language but just models so called entities that are mapped to a relational database. All operations are performed on the modeled objects.

The disadvantage of ORM is the loss of efficiency of performed operations. Most of the operations which has been written in SQL are executed faster than in the case of operations which has been written with the posted ORM. Converted inquiries does not always take the optimal form. Very often because of the standard feature of ORM some complex SQL statements are divided into many smaller ones. This causes the execution of unnecessary operations. This situation wouldn't take place if the programmer wrote the code in SQL.

The use of ORM besides the actual reflectivity of objects also reduces the amount of the written code, which in effect significantly speeds up the time of creation information systems. One of the most popular ORM systems is Hibernate for Java language and NHibernate or LINQ to SQL for C \#.ORM systems in which the developer maps databases models on his own are much more efficient than those that automatically generate code in a programming language based on the model of the database.

For REM systems the ORM mechanisms will have a very wide application because of the speed of software development with its use. Software development, in which a database access mechanism is based on ORM is much simpler, faster and more flexible for modifications. The disadvantage of ORM for REM systems is a decrease of database efficiency.

\subsection{Object-oriented database}

This chapter examines the implications of the possible use of object-oriented database in REM systems. 
Relational model, despite its popularity has, however, some shortcomings in the design and creation of more complex applications, such as systems for software engineering, industrial systems, telecommunications, multimedia, geographic information systems and systems for scientific experiments [7]. The requirements of new applications are different than traditional business systems. We need more complex structures describing objects, elongated transactions, new data types for storing images or large blocks of text, as well as the ability to define customoperations specific for concrete applications.

Relational databases are difficult to use in object-oriented programming languages, because of the necessity to use SQL statement, and then map the data to create an object model. So one of the reasons for the creation of object-oriented databases has been the growing popularity of object-oriented programming languages. Object-oriented database management systems have the following advantageous features: possibility to store of complex objects consistently, user-defined types, the identity of objects, relationships between objects, encapsulation, interfaces to objects, types, and/or class inheritance hierarchy and computational completeness.

A consortium of several producers of object databases called ODMG (Object Data Management Group) has proposed a standard for object-oriented databases, which resulted in a significant development of technology. It includes elements such as an object model based on Object Definition Language - ODL, and Object Query Language OQL [7]. After the liquidation of ODMG in 2001 the Object Management Group (OMG) was established, which is preparing the next generation of standard for object-oriented databases.

For REM systems, one of the most important features of object-oriented databases is the ability to create their own structure of objects and operations to be performed on those objects. Documents relating to real estate (property descriptions, texts transactions) can be more easily stored as objects. Similarly, images (pictures) of the property can be easily stored as objects.

At these objects, you can easily perform operations like searching (eg. relevant transactions or similar property). These operations can be performed without any intermediate phases transforming data between relational and object-oriented models. This is the basic advantage of object-oriented database systems from the point of view of REM systems.

But there are many reasons for limiting the possibility to use object-oriented database in REM systems. They are currently restrictions on most systems. The reasons are following:

- immaturity of technology - due to the risk of new technology, object oriented databases are used in smaller projects,

- instability of the producers - there is a lack of large producers who can ensure the stability of the operation of systems for many years,

- lack of qualified staff in the use of object-oriented databases,

- the high cost of conversion to a new data model. 


\subsection{Object-relational database}

This section presents the characteristics of object-relational databases as a compromise between the relational and object-oriented databases.

Conversion of relational database systems to object-oriented systems in existing systems would entail huge costs. Reaching a compromise, a new type of database has been created, namely object-relational databases. In an object-relational databases the benefits of both approaches has been combined: object-oriented and relational. The greatest short coming of relational database for REM systems i.e. earlier described range restriction was abolished in the new type of database. Architecture of object-relational databases can create your own non-primitive types, including object types and collections. The result is that actual objects in the databaseis represented inmuch easier way.

Object-relational databases are becoming increasingly popular. Members recognize the limitations of relational databases. However, the transition to object-oriented database is connected with the need to convert the data to the new model. It takes a lot of time and money. Therefore, most organizations that have recognized the advantages of object-oriented databases convert their databases to an object-relational databases, which does not require complicated conversion and reduce costs.

Object-relational databases can be used for REM systems.

However, the use of object-relational databases, as in the case of object-oriented database, is risky because of the young technology. There is not so much of technical support as in the case of relational databases.

\subsection{NoSQL - Not only SQL}

This chapter describes and characterizes the features of the latest types of databases such as NoSQL, which are beneficial for the REM system.

The origin of so called NoSQL movement, and then a new kind of database is derived from the demand of user access to information systems that are stored in the growing data sets. More and more institutions have sets of data, the number of which is calculated in GB, $\mathrm{TB}$, and sometimes PB (petabytes). Number of data means that the problem of the collection becomes a complicated issue [8].

The implementation of this object resulted in resignation from some properties of relational databases, among others transaction processing and tables joining, which according to their creators slowed down operations of processing large data sets. The functioning of thetransaction mechanism carries a large overhead of time what was the main reason of its resignation. The data in NoSQL databases is usually stored in JSON format in dynamic structures that are created at the time of recording the object to the base [5]. Customizing databases to contemporary problems caused the resignation from the mechanisms that have been developed over many years and were a great scientific achievement of different circles and institutions. In addition, the acceleration of database action is achieved by simplifying the structure of the data collection. So far, the data has been collected in the tables. NoSQL 
introduces a new type of structures that are based on a pair of "key-value" accordingly expandable depending on the type of database. NoSQL databases process data faster than relational databases [3].

You could say that NoSQL is more applicable to problems that are not standardized, i.e.thestructure of data stored in the database is not known a priori. This is how the REM systems look like and from this point of view, NoSQL databases are beneficial for REM systems.

In the REM systems a large number of data will be stored in the M-A type of systems. NoSQL databases places the problem of speed processing of large amounts of data at the highest level. It is also an advantage for the M-A system.

Resignation from the main advantages of relational databases, i.e. saving of the third normal form, causes the phenomenon of redundancy. On some systems, this can cause problems with the processing of data and the integrity of the stored data. Also for REE systems, this feature is not favorable due to the fact that these systems are used to prepare the valuation of property and lack of integrity of the data could lead to significant errors in the valuation.

The situation is different in the M-A systems. The lack of links between tables significantly facilitates horizontal scaling solutions developed on the basis of NoSQL. This is an important advantage for systems that collect a large amount of data, i.e. the M-A systems. Because the system has a database of documents intended only for reading or writing new data therefore the lack of integrity will not cause thesignificant errors for user.

Resignation from the mechanismof transactionscauses that the consistency of large amountof data collected in the database is exposed to high risk. REE systems that solve complex problems should be protected against improper modifications.

NoSQL databases are relatively young databases, the largest growth occurred in the past few years. Short time of bases functioning causes that they are not secured yet sufficiently. The data security is also affected by the fact that they are mostly developed in the open source licenses, resulting in the fact that the support systems are not on the same level as in commercial databases that have been operating for many years and have a large number of customers.

\section{Selection of a database type for the analyzed problem}

The chapter is a summary of the characteristics of the analyzed databases for REM systems and presents a selection of the relevant database. It is significant that for both types of systems (REE and M-A), we can choose different database.

The authors propose the following selection of databases:

- for the M-A system NoSQL database type,

- for REE system object-relational or object-oriented database.

Below we show the justification for this choice. 
Let's start with the M-A system. The most important requirements concern flexible structure of stored information and the possibility of high-speed processing of large amounts of data. NoSQL database meets both of these requirements according to the authors. The M-A system will be used at the same time even by a few hundred users. Easy horizontal scaling is a remarkable advantage of NoSQL databases for M-A systems. Let us notice that the features such as integrity and transaction mechanism are not a priority for the M-A systems. The only disadvantage of the NoSQL databases the young age of technology what may cause in the future problems obtaining technical support. It should be noted that the most popular systems that use NoSQL databases, i.e. Google, Amazon, LinkedIn, Facebook, Twitter, [9] use their own solutions dedicated to their problems - they are not dependent on the company providing the software.

Let us consider the REE system. The most important requirements for them concern the flexible structure of recording the information, consistency and integrity of stored data and transaction mechanism. These requirements best meet the object-oriented databases and object-relational databases. We should pay attention to ease of modeling objects in object-oriented and object-relational databases that can be created according to their real characters opposed to the relational databases. The relational database structure with complex problems is very complicated. It should be noted that the structure of data in REE systems is expanded and is expected to change over time. Thus, the database must be flexible due to changes in the data structure. Another advantage of object-oriented databases is faster speed in comparison with the relational database. NoSQL base type has been selected for the REE system due to the lack of transaction mechanism and the integrity of stored data.

\section{Conclusions}

This paper presents the task of selecting the type of database for the real estate market information systems. As a preliminary point, a general description of the real estate expert work was presented and on this basis the fundamental tasks for an information system supporting the work of an expert were defined. After the analysis, a concept of two different systems was proposed, which the real estate expert will benefit from. These include: monitoring and analysis of the global real estate market (M-A system) and real estate expert system (REE system), which will be used to analyze the local market and property valuation in the market. They differ in their architecture and the terms of reference. The data that will be stored in the databases of both systems has been characterized.

The following chapters analyzed different types of databases that can be potentially used to create these kinds of systems. The analysis have covered: relational databases, object-oriented databases, object-relational databases and a NoSQL databases. For M-A system NoSQL type of database was proposed, whereas for the REE system object-relational databases or object databases. 
The creation of a real system requires both IT knowledge and theoretical knowledge and practical experience in the operation area of ??the real estate market. The most difficult is to acquire knowledge of practical experience in this area, as in most cases the real estate experts are not interested in cooperation in creating informationsystems. The system is established only on the basis of theoretical knowledge, which would not be useful in practice. This is precisely the most important reason for the lack of professional systems on the market.

The co-author gained the necessary knowledge of the real estate market analysis and of real estates valuation, creating a prototype of the system in close cooperation with the experts of the real estate market.

\section{References}

[1] Król D., Lasota T., Nalepa W., Trawiński B., Fuzzy system model to assist with real estate appraisals. In 20th International Conference on Industrial, Engineering and Other Applications of Applied Intelligent Systems, IEA/AIE 2007, Kyoto, Japan. LNCS/LNAI. vol. 4570, 2007, pp. 260-269.

[2] Dudek-Dyduch E., Rączka K., Information system for real estate valuation. Automatyka (AGH), t. 15, z. 3, 2011, pp. 637-652.

[3] Leavitt N., Will NoSql databases live up to their promise? Computer, vol. 43, No. 2, 2010, pp. $12-14$.

[4] Tudorica B.G., Bucur C., A comparison between several NoSQL databases with comments and notes. 10th Roedunet International Conference (RoEduNet), 2011.

[5] Boicea A., Radulescu F., Agapin L.I., MongoDB vs Oracle - database comparison. Third International Conference on Emerging Intelligent Data and Web Technologies, 2012.

[6] Bonnet L., What NoSQL Can Do for Data Aggregation and BI in Large Repositories. Database and Expert Systems Applications (DEXA), 2011 22nd International Workshop, Conference Publications, pp. 483-488.

[7] Stones R., Mathew N., Beginning Databases: From Novice to Professional. 2005, Apress.

[8] Jing Han, Haihong E., Guan Le, Jian Du, Survey on NoSQL database. Pervasive Computing and Applications (ICPCA), 6th International Conference, 2011.

[9] Indrawan-Santiago M., Database Research: Are We At A Crossroad? Reflection on NoSQL, 2012 15th International Conference on Network-Based Information Systems.

[10] Orend K., Analysis and Classification of NoSQL Databases and Evaluation of their Ability to Replace an Object-relational Persistence Layer. Master Thesis - Technische Universitat Munchen, 2010.

[11] Bonnet L., Laurent A., Sala M., Laurent B., Sicard N., Reduce, You Say: What NoSQL Can Do for Data Aggregation and BI in Large Repositories. 22nd International Workshop on Database and Expert Systems Applications, 2011.

[12] Arslan A., Yilmazel O., Quality Benchmarking Relational Databases and Lucene in the TREC4 Adhoc Task Environment. Computer Science and Information Technology (IMCSIT), Proceedings of the 2010 International Multiconference, 2010.

[13] Peng Xiang, Ruichun Hou, Zhiming Zhou, Cache and consistency in NOSQL. Computer Science and Information Technology (ICCSIT), 3rd IEEE International Conference, 2010.

[14] Konowalczuk J., Ramian T., Informational needs and barriers of real estate market from the perspective of real estate valuation in Poland. Journal of the Polish Real Estate Scientific Society, vol. 20, No. 2, 2012. 
[15] Kucharska-Stasiak E., Value measurement in economics-consequences for real estate valuation. Journal of the Polish Real Estate Scientific Society, vol. 19, No. 1, 2011.

[16] Budzyński T., Methodology of the update of prices on the example of house real estates. Journal of the Polish Real Estate Scientific Society, vol. 18, No. 1, 2010.

[17] Radzewicz A., Wiśniewski R., Variables, features and attributes of real estate. Journal of the Polish Real Estate Scientific Society, vol. 20, No. 1, 2010.

[18] http://www.pronet.com.pl

[19] http://www.obrn.pl

[20] http://www.wycenter.pl 